\title{
The Correlation Structure of the Output of an ATM Multiplexer
}

\author{
C. Blondia and F. Geerts \\ Performance Analysis and \\ Telecommunication Systems Research Group \\ Dept. Mathematics and Computer Science \\ University of Antwerp \\ Universiteitsplein, 1, B-2610 Antwerp-Belgium \\ blondia@uia.ua.ac.be-fgeerts@luc.ac.be
}

\begin{abstract}
In this paper we study the correlation structure of the output process of an ATM multiplexer. We consider two special cases : (i) the output process of the D-BMAP/D/1/N queue, a generic model for an ATM multiplexer and (ii) a process which results from a renewal process which shares the output link of a multiplexer with other connections. Both output processes belong to the versatile class of discrete-time Markovian arrival processes (D-MAP's). We derive an expression for the Index of Dispersion for Counts (IDC) and for the Index of Dispersion for Intervals (IDI) of a D-MAP. Two classes of DMAP's are considered depending on the eigenvalues of the transition matrix : those with an aperiodic transition matrix and those with a periodic transition matrix. For both cases we derive a closed form formula for the limit of the IDC (which coincides with the limit of the IDI) and for the convergence rate of the covariance sequence. These results are then applied to the two special cases of output processes.
\end{abstract}

\section{Keywords}

ATM, Correlation, D-MAP, IDC, IDI, multiplexer

\section{INTRODUCTION}

The Asynchronous Transfer Mode (ATM) must contribute to an efficient use of the network resources while guaranteeing the required Quality of Service of the different traffic streams. To quantitatively study these goals of ATM, there has been made a considerable effort to model ATM traffic sources together with the different network elements. The basic queueing model for these studies is a multiplexer whose input consists of a superposition of ATM traffic sources. Several approaches have been used to derive the required performance measures of such a multiplexer (e.g. fluid flow, matrix-analytical,

The original version of this chapter was revised: The copyright line was incorrect. This has been corrected. The Erratum to this chapter is available at DOI: 10.1007/978-0-387-35353-1_28 
generating functions, etc...). These models are valid at the entrance of the ATM network, but may be inadequate as generic traffic model for e.g. endto-end delay studies.

To analyze a whole path in an ATM network analytically, modeling and characterizing the output process of an ATM multiplexer is an essential step. This output process will become input process, together with external sources, of the next network element. In addition, a characterization of the output process allows an evaluation of the smoothing effect of bursty traffic when passing through a multiplexer.

The main problem when using the output process of the previous stage as input to the next stage is that after a few stages the resulting process becomes very complicated and hence intractable. Therefore, it is necessary to capture its most significant characteristics. Two very important properties are the correlation between the number of cells in the output process in successive slots and the correlation between interdeparture times. Many studies (Heffes \& Lucantoni 1986, Sriram \& Whitt 1986) have confirmed the impact of the autocovariance sum on the queueing performance. In particular these studies stress the importance of the Index of Dispersion for Counts (IDC) and the Index of Dispersion for Intervals (IDI), together with their limits.

In this paper we study a particular class of output processes, namely the discrete-time Markovian arrival processes (D-MAP's). This choice is motivated by two important special cases.

(i) In previous papers (Blondia \& Casals 1992, Blondia 1993), it has been shown that the D-MAP is a generic model for ATM traffic, since the output process of a multiplexer whose input consists of a superposition of D-MAP's is again a D-MAP.

(ii) Assume that a tagged ATM connection, modeled by means of a process with renewal cell interarrival time distribution, shares a multiplexer with other connections (modeled as a batch process with renewal batch size distribution). The tagged connection belongs, after passing through the multiplexer, to the class of D-MAP's. More details can be found in (Blondia \& Casals 1996).

For the class of D-MAP's, we study the covariance of the interdeparture time, the covariance of the number of departures in a slot, the IDC, the IDI and their limits.

We distinguish two classes of D-MAP's : those with aperiodic transition matrix and those whose transition matrix is periodic. These two classes naturally arise from the two examples of output processes considered in this paper. Other examples of the use of periodic D-MAP's as well as some properties of their correlations can be found in (Herrmann 1994a, Herrmann 1994b).

The paper is organized as follows. Section 2 recalls the definition of the discrete-time Markovian arrival process and some related notions. We discuss the two important examples of D-MAP's which are models for the output processes discussed above. In Section 3, we investigate the correlation structure of the number of arrivals in a slot of a D-MAP. In Section 4, the correlation 
structure of the interarrival times of a D-MAP are investigated. We also derive expressions for the limit of the IDC and the IDI. These results are then applied to numerical examples in Section 5. Conclusions are drawn in Section 6.

\section{A MODEL FOR THE OUTPUT OF AN ATM MULTIPLEXER}

In this section we identify two important models for output processes of an ATM multiplexer. Both these examples belong to a class of versatile Markovian processes, called D-MAP's. First we recall the definition of this class of processes.

\subsection{A Discrete-time Markovian Arrival Process (D-MAP)}

We recall the definition of the D-BMAP, a batch Markovian arrival process which has proven its usefulness in many papers (Blondia \& Casals 1992, Blondia 1993, etc ...). This process is the discrete-time version of the MAP defined in (Lucantoni 1991), which was originally called N-Process in (Neuts 1979).

Consider a discrete-time Markov chain with transition matrix D. Suppose that at time $k$ this chain is in some state $i, 1 \leq i \leq m$. At the next time instant $k+1$, there occurs a transition to another or possible the same state and a batch arrival may or may not occur. With probability $\left(d_{0}\right)_{i, j}, 1 \leq i \leq m$, there is a transition to state $j$ without an arrival, and with probability $\left(d_{n}\right)_{i, j}$, $1 \leq i \leq m, n \geq 1$, there is a transition to state $j$ with a batch arrival of size $n$. We have that

$\sum_{n=0}^{\infty} \sum_{j=1}^{m}\left(d_{n}\right)_{i, j}=1$.

Clearly the matrix $\mathbf{D}_{0}$ with elements $\left(d_{0}\right)_{i, j}$ governs transitions that correspond to no arrivals, while the matrices $\mathbf{D}_{n}$ with elements $\left(d_{n}\right)_{i, j}, n \geq 1$, govern transitions that correspond to arrivals of batches of size $n$.

The matrix $\mathbf{D}=\sum_{n=0}^{\infty} \mathbf{D}_{\mathbf{n}}$ is the transition matrix of the underlying Markov chain . Let $\bar{\pi}$ be stationary probability vector of this Markov process, i.e. $\overline{\boldsymbol{\pi}} \mathbf{D}=\overline{\boldsymbol{\pi}}, \quad \overline{\boldsymbol{\pi}} \overline{\mathbf{e}}=1$, where $\overline{\mathbf{e}}$ is a column vector of 1 's.

The fundamental arrival rate $\lambda$ of this process is given by $\lambda=\bar{\pi}\left(\sum_{k=1}^{\infty} k \mathbf{D}_{k}\right) \overline{\mathbf{e}}$. A D-MAP is a special case of a D-BMAP, where arrivals have a batch of size 1. For examples we refer to (Blondia 1993).

We recall some results concerning D-MAP's which are needed in the sequel of this paper. A D-MAP is characterized by means of its two matrices $\mathbf{D}_{\mathbf{0}}$ and $\mathbf{D}_{\mathbf{1}}$. Let $\overline{\boldsymbol{\pi}}$ be the steady state vector of the underlying Markov chain 
$\mathbf{D}_{\mathbf{0}}+\mathbf{D}_{\mathbf{1}}$. The fundamental arrival rate $\lambda$ of this process is now given by $\lambda=\bar{\pi} \mathbf{D}_{\mathbf{1}} \overline{\mathbf{e}}$. Observe the phase of the process at arrival instants. The phase transition matrix between these instants is given by $\left(\mathbf{I}-\mathbf{D}_{\mathbf{0}}\right)^{-1} \mathbf{D}_{\mathbf{1}}$.

Let $\overline{\mathbf{p}}$ be the stationary vector of the phase at arrival instants, i.e.

$\overline{\mathbf{p}}\left(\mathbf{I}-\mathbf{D}_{\mathbf{0}}\right)^{-1} \mathbf{D}_{\mathbf{1}}=\overline{\mathbf{p}}, \quad \overline{\mathbf{p}} \overline{\mathbf{e}}=1$.

This vector can be expressed in terms of $\overline{\boldsymbol{\pi}}$ as follows : $\overline{\mathbf{p}}=\frac{1}{\lambda} \overline{\boldsymbol{\pi}} \mathbf{D}_{\mathbf{0}}$. Two special examples of a D-MAP which will be studied in the next subsections.

\subsection{The Output Process of the D-BMAP/D/1/N Queue}

In (Blondia 1993), it has been shown that the output process of a queue of type D-BMAP $/ \mathrm{D} / 1 / \mathrm{N}$ belongs to the class of D-MAP's. Indeed, let the input process be defined by the matrices $\mathbf{D}_{\mathbf{n}}, n \geq 0$. Then the output process is a D-MAP with parameters

$$
\mathbf{D}_{0}=\left(\begin{array}{llll}
\mathbf{D}_{0} & \cdots & \mathbf{D}_{N-1} & \sum_{i=N}^{\infty} \mathbf{D}_{i} \\
0 & \cdots & 0 & 0 \\
\vdots & \ddots & \vdots & \vdots \\
0 & \cdots & 0 & 0 \\
0 & \cdots & 0 & 0 \\
0 & \cdots & 0 & 0
\end{array}\right), \mathbf{D}_{1}=\left(\begin{array}{lllll}
0 & 0 & \ldots & 0 & 0 \\
\mathbf{D}_{0} & \mathbf{D}_{1} & \cdots & \mathbf{D}_{N-1} & \sum_{i=N}^{\infty} \mathbf{D}_{i} \\
0 & \mathbf{D}_{0} & \cdots & \mathbf{D}_{N-2} & \sum_{i=N}^{\infty} \mathbf{D}_{i} \\
\vdots & \vdots & \vdots & \vdots & \vdots \\
0 & 0 & \cdots & \mathbf{D}_{1} & \sum_{i=1}^{\infty} \mathbf{D}_{i}
\end{array}\right)
$$

The process has been studied in detail in (Blondia \& Casals 1996), e.g. the distribution of the length of $k$ consecutive interdeparture times, the busy period distribution and the interdeparture time distribution are derived there.

\subsection{A Renewal Process Mixed with Background Traffic}

We tag a connection which shares the output link of a multiplexer with other connections, and describe this tagged connection after passage through the multiplexer (See Figure 1). First we show that under certain conditions the resulting process belongs to the class of D-MAPs. Details can be found in (Blondia \& Casals 1996).

We consider a discrete-time queueing system with deterministic service time, the duration of which equals one time-unit (i.e. a time-slot). We assume the queue has a finite capacity of $N$ cells.

The input traffic consists of 2 streams :

\section{- Tagged traffic stream}

This stream has a cell interarrival time distribution which is assumed to be renewal, defined by the vector $\overline{\mathbf{b}}=\left(b_{1}, \ldots, b_{K}\right)$, where

$b_{k}=\operatorname{Pr}\{$ interarrival time between consecutive cells is $k$ slots $\}$. 


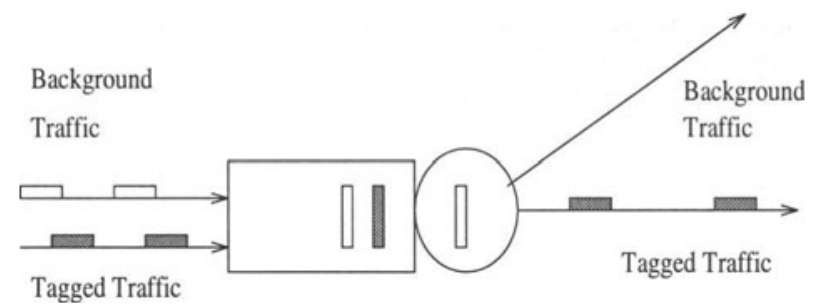

Figure 1 Queueing model for departure process of tagged stream

Examples :

(i) CBR traffic (interarrival time is deterministic) ;

(ii) On/off sources, where the on and off periods are assumed to have a duration which is geometrically distributed and such that while in the on period, the cell interarrival time is deterministic.

- Background traffic

The number of background cells arriving in a time slot is a renewal process; let

$a_{k}=\operatorname{Pr}\{k$ arrivals during a time slot $\}, k \geq 0$.

Example :

Poisson background traffic with arrival rate $\lambda, a_{k}=\frac{\lambda^{k}}{k !} e^{-\lambda}, k \geq 0$.

In order to describe the tagged traffic stream, after it has left the multiplexer which it shares with background traffic, we introduce the matrices $\mathbf{H}_{1}$ and $\mathrm{H}_{2}$.

The transition matrix of the number of cells in the system (between consecutive slots) when only the background stream is taken into account is given by

$$
\mathbf{H}_{1}=\left(\begin{array}{llllll}
a_{0} & a_{1} & a_{2} & \ldots & a_{N-1} & \sum_{n=N}^{\infty} a_{n} \\
a_{0} & a_{1} & a_{2} & \ldots & a_{N-1} & \sum_{n=N}^{\infty} a_{n} \\
0 & a_{0} & a_{1} & \ldots & a_{N-2} & \sum_{n=N-1}^{\infty} a_{n} \\
\cdot & \cdot & \cdot & . & . & \cdot \\
\cdot & \cdot & . & . & . & . \\
0 & 0 & 0 & \ldots & a_{0} & \sum_{n=1}^{\infty} a_{n}
\end{array}\right) .
$$


The transition matrix of the number of cells in the queueing system between consecutive slots, when both streams are taken into account and knowing that an arrival of the tagged stream occurs, is given by

$$
\mathbf{H}_{2}=\left(\begin{array}{llllll}
0 & a_{0} & a_{1} & \ldots & a_{N-2} & \sum_{n=N-1}^{\infty} a_{n} \\
0 & a_{0} & a_{1} & \ldots & a_{N-3} & \sum_{n=N-2}^{\infty} a_{n} \\
0 & 0 & a_{0} & \ldots & a_{N-4} & \sum_{n=N-3}^{\infty} a_{n} \\
. & . & . & . & . & \cdot \\
. & . & . & . & . & . \\
0 & 0 & 0 & \ldots & 0 & 1
\end{array}\right)
$$

Now we give an important property of the interdeparture time of consecutive cells of the tagged stream. The proof is straightforward.

Property 1 Assume that two consecutive arrivals of the tagged stream observe a queue length of $i_{1}$, resp. $i_{2}$. Then the interdeparture time between these two cells of the tagged stream is $k-i_{1}+i_{2}$, with probability $b_{k}, 1 \leq k \leq K$.

We show that the tagged stream after the multiplexer is a D-MAP.

Define for each slot $k$ the following variables :

- $s_{k}$ : number of slots to go until the next departure of the tagged stream. Clearly, its value is the interdeparture time when a cell of the tagged stream leaves the multiplexer and it decreases by 1 at each slot, until it reaches the value 0 (i.e. when the next departure occurs) $\left(s \leq s_{k} \leq S=N+K-1\right)$.

- $i_{k}$ : queue length of the multiplexer at the arrival instant of a tagged cell, which was the last of the tagged stream to depart before slot $k$. This means that the value of $i_{k}$ remains constant between departures of the tagged stream $\left(1 \leq i_{k} \leq N\right)$.

Then $\left\{\left(s_{k}, i_{k}\right) \mid k \geq 0\right\}$ forms a discrete-time Markov chain, with the following transition matrix $\mathbf{D}$.

- If $s_{k}=s>0$ and $1 \leq i_{k}=i \leq N$,

$$
\begin{aligned}
\mathbf{D}_{(s, i),(s-1, i)} & =1 \\
& =0 \quad \text { elsewhere, }
\end{aligned}
$$

- If $s_{k}=s=0$ and $1 \leq i \leq N$ (i.e. a tagged cell departed form the multiplexer at slot $k$ ):

$$
\begin{aligned}
\mathbf{D}_{(0, i),(k-i+j-1, j)} & =b_{k} \times\left[\mathbf{H}_{2} \mathbf{H}_{1}^{k-1}\right]_{i+1, j+1}, k=1, \cdots, K, \quad j=1, \cdots, N \\
& =0 \text { elsewhere. }
\end{aligned}
$$


The matrix $\mathbf{D}$ defined in this way is the transition matrix of this Markov chain. The matrices $\mathbf{D}_{\mathbf{0}}$ and $\mathbf{D}_{\mathbf{1}}$ defined as

$$
\left[\mathbf{D}_{0}\right]_{s, i}=\left\{\begin{array}{ll}
{[\mathbf{D}]_{s, i}} & \forall(s, i), s \neq 0 \\
0 & \forall(0, i)
\end{array} \quad \text { and } \quad\left[\mathbf{D}_{\mathbf{1}}\right]_{s, i}= \begin{cases}{[\mathbf{D}]_{0, i}} & \forall(0, i) \\
0 & \forall(s, i), s \neq 0\end{cases}\right.
$$

describe the cell generation process of the D-MAP that models the departure process of the tagged traffic stream.

Hence under the assumption that cells of both tagged and background connections arrive according to independent renewal processes, the output process of the tagged traffic stream belongs to the class of D-MAP's.

The transition matrix has the following form

$\mathbf{D}=\left(\begin{array}{llllll}\mathbf{U}_{0} & \mathbf{U}_{1} & \mathbf{U}_{2} & \ldots & \mathbf{U}_{S-1} & \mathbf{U}_{S} \\ \mathbf{I} & \mathbf{0} & \mathbf{0} & \ldots & \mathbf{0} & \mathbf{0} \\ \mathbf{0} & \mathbf{I} & \mathbf{0} & \ldots & \mathbf{0} & \mathbf{0} \\ \vdots & \vdots & \vdots & \ddots & \vdots & \vdots \\ \mathbf{0} & \mathbf{0} & \mathbf{0} & \ldots & \mathbf{I} & \mathbf{0}\end{array}\right)$

In view of the special form of $\mathbf{D}$, it is possible to simplify the computations as follows.

First the steady state vector $\overline{\boldsymbol{\pi}}=\left(\overline{\boldsymbol{\pi}}_{0}, \overline{\boldsymbol{\pi}}_{1}, \ldots, \overline{\boldsymbol{\pi}}_{S}\right)$ of the matrix $\mathbf{D}$ is computed.

As $\bar{\pi}$ satisfies $\bar{\pi} \mathbf{D}=\bar{\pi}$, we have that

$\overline{\boldsymbol{\pi}}_{0} \mathbf{U}_{i-1}+\overline{\boldsymbol{\pi}}_{i}=\overline{\boldsymbol{\pi}}_{i-1}, \quad i=1, \ldots, S$

$\bar{\pi}_{0} \mathbf{U}_{S}=\bar{\pi}_{S}$.

This implies that $\bar{\pi}_{0}$ satisfies $\bar{\pi}_{0}\left(\sum_{i=0}^{S} \mathbf{U}_{i}\right)=\bar{\pi}_{0}$. But as $\sum_{i=0}^{S} \mathbf{U}_{i}=\mathbf{H}=$ $\sum_{k=1}^{K} b_{k} \mathbf{H}_{2} \mathbf{H}_{1}^{k-1}$, we have that

$\bar{\pi}_{0} \mathbf{H}=\bar{\pi}_{0}$

Furthermore, as $\sum_{i=0}^{S} \bar{\pi}_{i} \overline{\mathbf{e}}=1$, we derive that

$\bar{\pi}_{0}\left[\sum_{i=0}^{S}(i+1) \mathbf{U}_{i}\right] \overline{\mathbf{e}}=1$

Formulas (1) and (2) completely determine $\bar{\pi}_{0}$. The other $\bar{\pi}_{i}$ 's are given by $\overline{\boldsymbol{\pi}}_{n}=\overline{\boldsymbol{\pi}}_{0}\left(\sum_{i=n}^{S} \mathbf{U}_{i}\right)$. 


\section{CORRELATION STRUCTURE OF THE NUMBER OF ARRIVALS OF A D-MAP}

In this section we refer to the occurance of a cell in a slot as to a cell arrival (since the D-MAP is called Markovian arrival process), but the results will be applied in the next section to D-MAP's which are departure processes. We study the correlation between both successive interarrival times and number of arrivals in successive slots.

Recall that when $\left(X_{1}, X_{2}, \ldots, X_{k}\right)$ are random variables, then the correlation between $X_{1}$ and $X_{k}$ can be expressed in terms of the covariance matrix

$\operatorname{cov}\left(X_{1} X_{k}\right)=\mathbf{E}\left[\left(X_{1}-\mu_{1}\right)\left(X_{k}-\mu_{k}\right)\right]$

with $\mu_{1}$ and $\mu_{k}$ being the scalar mean of $X_{1}$ and $X_{k}$.

The scalar covariance function is given by

$\operatorname{cov}\left(X_{1} X_{k}\right)=\bar{\pi} \operatorname{cov}\left(X_{1} X_{k}\right) \overline{\mathbf{e}}=\bar{\pi} \mathbf{E}\left[X_{1} X_{k}\right] \overline{\mathbf{e}}-\mu_{1} \mu_{k}$

\subsection{Correlation between Arrivals}

In this subsection we study the correlation between the number of arrivals in a slot.

Let $\left(X_{1}, \ldots, X_{k}\right)$ be random variables, where $X_{i}$ is the number of arrivals $(0$ or 1) at time slot $i$. In (Blondia \& Theimer 1989), it has been shown that

Theorem 1 The scalar covariance of $X_{1}$ and $X_{k}$ is given by

$$
\begin{aligned}
& \operatorname{cov}\left(X_{1} X_{1}\right)=\bar{\pi} \mathbf{D}_{\mathbf{1}} \overline{\mathbf{e}}-\left(\bar{\pi} \mathbf{D}_{\mathbf{1}} \overline{\mathbf{e}}\right)^{2} \\
& \operatorname{cov}\left(X_{1} X_{k+1}\right)=\bar{\pi} \mathbf{D}_{\mathbf{1}}\left(\mathbf{D}_{\mathbf{0}}+\mathbf{D}_{\mathbf{1}}\right)^{k-1} \mathbf{D}_{\mathbf{1}} \overline{\mathbf{e}}-\left(\overline{\boldsymbol{\pi}} \mathbf{D}_{\mathbf{1}} \overline{\mathbf{e}}\right)^{2}, \quad k \geq 1 .
\end{aligned}
$$

Remark that an extension of this theorem for D-BMAP's was obtained in (Blondia 1993).

\section{(a) Correlation of an aperiodic D-MAP}

In order to identify over what time period correlations do exist, we study the way the sequence of covariances $\operatorname{cov}\left(X_{1} X_{k}\right)$ converges to its limiting value (if it exists).

We have to distinguish between two cases depending on the number of eigenvalues of $\mathbf{D}_{\mathbf{0}}+\mathbf{D}_{\mathbf{1}}$ on the unit-circle.

Theorem 2 Assume that the matrix $\mathbf{D}_{\mathbf{0}}+\mathbf{D}_{\mathbf{1}}$ is diagonizable and that the 
eigenvalue 1 is the only one on the unit circle. The convergence of the covariance of the number of arrivals towards zero is geometric, The ratio is determinded by the eigenvalue $\lambda_{2}$ of $\mathbf{D}_{0}+\mathbf{D}_{1}$ with the largest absolute value, excluding 1. Thus

$\left|\operatorname{cov}\left(X_{1}, X_{k}\right)\right|=c \cdot\left|\lambda_{2}\right|^{k}$, for $k \rightarrow \infty$,

with $c$ a certain constant.

PROOF. Since $\mathbf{D}_{\mathbf{0}}+\mathbf{D}_{\mathbf{1}}$ is diagonalizable, we can find the following spectral representation (see e.g. (Cinlar 1975), Theorem 5.1, p.379)

$\mathbf{D}_{\mathbf{0}}+\mathbf{D}_{\mathbf{1}}=\mathbf{B}_{1}+\lambda_{2} \mathbf{B}_{2}+\cdots+\lambda_{n} \mathbf{B}_{n}$

From the theory of Perron-Frobenius we know that an irreducible stochastic matrix $\mathbf{D}_{\mathbf{0}}+\mathbf{D}_{\mathbf{1}}$ has a unique eigenvalue 1 and the other eigenvalues have absolute value $|\lambda|<1$.

Substituting this representation for $\mathbf{D}_{\mathbf{0}}+\mathbf{D}_{\mathbf{1}}$ in the expression for $\operatorname{cov}\left(X_{1}, X_{k}\right)$ derived in Theorem 1, yields

$\operatorname{cov}\left(X_{1}, X_{k}\right)=\lambda_{2}^{k-1} \bar{\pi} \mathbf{D}_{1} \mathbf{B}_{2} \mathbf{D}_{\mathbf{1}} \overline{\mathbf{e}}+\cdots+\lambda_{n}^{k-1} \bar{\pi} \mathbf{D}_{1} \mathbf{B}_{n} \mathbf{D}_{\mathbf{1}} \overline{\mathbf{e}}$

and this implies the required result.

\section{(b) Correlation of a periodic D-MAP}

When there is more than one eigenvalue on the unit circle (apart from the eigenvalue 1), then the situation is somewhat more complicated. It means that the matrix $\mathbf{D}_{0}+\mathbf{D}_{1}$ is periodic, i.e. there exist $\delta>0$ (i.e. the the number of eigenvalues on the unit circle) and matrices $\mathbf{F}_{i}, i=1,2, \ldots, \delta$, such that $\mathbf{D}_{\mathbf{0}}+\mathbf{D}_{\mathbf{1}}$ can be transformed into

$\mathbf{D}_{\mathbf{0}}+\mathbf{D}_{1}=\left(\begin{array}{llllll}\mathbf{0} & \mathbf{F}_{1} & \mathbf{0} & \ldots & \mathbf{0} & \mathbf{0} \\ \mathbf{0} & \mathbf{0} & \mathbf{F}_{2} & \ldots & \mathbf{0} & \mathbf{0} \\ \vdots & \vdots & \vdots & \ddots & \vdots & \vdots \\ \mathbf{0} & \mathbf{0} & \mathbf{0} & \ldots & \mathbf{0} & \mathbf{F}_{\delta-1} \\ \mathbf{F}_{\delta} & \mathbf{0} & \mathbf{0} & \ldots & \mathbf{0} & \mathbf{0}\end{array}\right)$

The $\delta$ different eigenvalues with $\left|\lambda_{j}\right|=1$, are $\left\{\lambda_{j}=e^{\frac{2 \pi i}{\delta} j} \mid j=0,1, \ldots, \delta-1\right\}$. The convergence mentioned in Theorem 4 , is no longer valid. However it is possible to prove the following result. We denote by $\oplus$ addition modulo $\delta$.

Theorem 3 Assume that the matrix $\mathbf{D}_{\mathbf{0}}+\mathbf{D}_{\mathbf{1}}$ has $\delta$ eigenvalues on the unit 
circle, i.e. it has the form as shown in (3). Let $\bar{\pi}^{0}=\left(\bar{\pi}_{1}^{0}, \ldots, \bar{\pi}_{\delta}^{0}\right)$ (resp. $\left.\bar{\nu}^{0}=\left(\overline{\boldsymbol{\nu}}_{1}^{0}, \ldots, \overline{\boldsymbol{\nu}}_{\delta}^{0}\right)\right)$ the left (resp. right) eigenvector of $\mathbf{D}_{\mathbf{0}}+\mathbf{D}_{\mathbf{1}}$ with eigenvalue one.

If $\mu_{j}=\overline{\boldsymbol{\pi}}_{j}^{0} \mathbf{F}_{j} \overline{\boldsymbol{\nu}}_{j \oplus 1}^{0}$, then the $\delta$ possible limits of the $\delta$ subsequences of the covariance sequence are given by

$\lim _{n \rightarrow \infty} \operatorname{cov}\left(X_{1}, X_{n \delta+l}\right)=(\delta-1) \sum_{i=1}^{\delta} \mu_{i} \mu_{(i \oplus l)}-\sum_{1 \leq i, j \leq \delta} \mu_{j \neq i \oplus l} \mu_{j}, l=1,2, \cdots, \delta$.

PROOF. See (Geerts 1997).

From this theorem we see that in the covariance sequence we can distinguish $\delta$ subsequences each converging to a different limit. Note that some of these limiting values can be equal.

\subsection{Limit of Index of Dispersion for Counts of a D-MAP}

An important measure for the correlation is the Index of Dispersion for Counts (IDC).

Denote by $N_{i j}^{(k)}(l)$ the conditional probability that in $k$ slots there are $l$ arrivals and at the $k$-th slot the phase of the process is $j$, given that at time $\mathrm{t}=0$ the phase was $i$. Let

$\mathbf{N}^{(\mathbf{k})}(z)=\sum_{l=0}^{\infty} \mathbf{N}^{(\mathbf{k})}(l) z^{l}$.

Clearly $\mathbf{N}^{(\mathbf{k})}(z)=\left(\mathbf{D}_{\mathbf{0}}+\mathbf{D}_{\mathbf{1}} z\right)^{k}$. We define the index of dispersion for counts (IDC) as

$C(k)=\frac{\operatorname{Var}\left[N^{(k)}\right]}{\mathrm{E}\left[N^{(k)}\right]}$,

where $\operatorname{Var}\left[N^{(k)}\right]$ and $\mathrm{E}\left[N^{(k)}\right]$ denote the scalar variance, resp. scalar mean, of the variable $N^{(k)}$. For a general stationary arrival process, it is known that the following holds

$C(k)=\frac{k \operatorname{cov}\left(X_{1} X_{1}\right)+2 \sum_{j=1}^{k-1}(k-j) \operatorname{cov}\left(X_{1} X_{1+j}\right)}{k \mathrm{E}\left[X_{1}\right]}$.

It is also well known that for a process for which the number of arrivals in a slot is renewal, $C(k)=c_{1}^{2}$, for all $k \geq 1$, where $c_{1}^{2}$ is the squared coefficient of variation of the number of arrivals in a slot. In particular for a Bernoulli 
process, $C(k)=1-p$ (Sriram \& Whitt 1986), where $p$ is the probability of generating an arrival in a slot.

In contrast to the limit of the correlation of the arrival process of a D-MAP, the limit of the IDC is not dependent on the periodicity of the transition matrix $\mathbf{D}_{\mathbf{0}}+\mathbf{D}_{\mathbf{1}}$. The limit of the IDC has a unique value. We give an explicit expression of this limit for a DMAP in the next Theorem.

Theorem 4 Consider a D-MAP with ergodic Markov chain $\mathbf{D}_{\mathbf{0}}+\mathbf{D}_{\mathbf{1}}$. The limit of the IDC of this D-MAP is given by

$\lim _{k \rightarrow+\infty} C(k)=\frac{\bar{\pi} \mathbf{D}_{\mathbf{1}} \overline{\mathbf{e}}-\left[\bar{\pi} \mathbf{D}_{\mathbf{1}} \overline{\mathbf{e}}\right]^{2}+2 \bar{\pi} \mathbf{D}_{\mathbf{1}} \mathbf{Z} \mathbf{D}_{\mathbf{1}} \overline{\mathbf{e}}-2 \bar{\pi}\left[\mathbf{D}_{\mathbf{1}}\right]^{2} \overline{\mathbf{e}}}{\bar{\pi} \mathbf{D}_{\mathbf{1}} \overline{\mathbf{e}}}$,

with $\mathbf{Z}$ the fundamental matrix of the Markov chain $\mathbf{D}_{\mathbf{0}}+\mathbf{D}_{\mathbf{1}}$, given by

$\mathbf{Z}=\left[\mathbf{I}-\left(\mathbf{D}_{\mathbf{0}}+\mathbf{D}_{\mathbf{1}}-\overline{\mathbf{e}} \boldsymbol{\pi}\right)\right]^{-1}$

PROOF. In view of Theorem 1,

$$
\begin{aligned}
\sum_{j=1}^{k-1} \frac{k-j}{k} \operatorname{cov}\left(X_{1}, X_{1+j}\right) & =\sum_{j=1}^{k-1} \frac{k-j}{k}\left[\bar{\pi} \mathbf{D}_{\mathbf{1}}\left[\mathbf{D}_{\mathbf{0}}+\mathbf{D}_{\mathbf{1}}\right]^{j-1} \mathbf{D}_{\mathbf{1}} \bar{e}-\left(\bar{\pi} \mathbf{D}_{\mathbf{1}} \overline{\mathbf{e}}\right)^{2}\right] \\
& =\bar{\pi} \mathbf{D}_{\mathbf{1}}\left\{\sum_{j=1}^{k-1} \frac{k-j}{k}\left[\left(\mathbf{D}_{\mathbf{0}}+\mathbf{D}_{\mathbf{1}}\right)^{j-1}-\overline{\mathbf{e}} \pi\right]\right\} \mathbf{D}_{\mathbf{1}} \overline{\mathbf{e}}
\end{aligned}
$$

In view of (Kemeny \& Snell 1967, Theorem 5.1 .4 ,p.101), the following series is Cesaro-summable

$\sum_{j=1}^{k-1} \frac{k-j}{k}\left[\left(\mathbf{D}_{0}+\mathbf{D}_{1}\right)^{j-1}-\overline{\mathbf{e}} \boldsymbol{\pi}\right]$

and the Cesaro-limit is given by $\mathbf{Z}-\mathbf{I}$, with $\mathbf{Z}=\left[\mathbf{I}-\left(\mathbf{D}_{\mathbf{0}}+\mathbf{D}_{\mathbf{1}}-\overline{\mathbf{e}} \boldsymbol{\pi}\right)\right]^{-1}$ the fundamental matrix of the Markov chain $\mathbf{D}_{\mathbf{0}}+\mathbf{D}_{1}$. From this we obtain expression (4).

\section{CORRELATION STRUCTURE OF INTERARRIVAL TIMES OF A D-MAP}

Let $\left(T_{1}, \ldots, T_{k}\right)$ be random variables representing the interarrival times until the $k$-th arrival. Based on the results in (Blondia \& Theimer 1989) it is straightforward to prove the following expression for the scalar covariance of $T_{1}$ and $T_{k}, k \geq 1$. 
Theorem 5 The scalar covariance of $T_{1}$ and $T_{k}$ is given by the following formulas :

$$
\begin{aligned}
& \operatorname{cov}\left(T_{1} T_{1}\right)=2 \overline{\mathbf{p}}\left[\left(\mathbf{I}-\mathbf{D}_{\mathbf{0}}\right)^{-1}\right]^{2} \overline{\mathbf{e}}-\left[\overline{\mathbf{p}}\left(\mathbf{I}-\mathbf{D}_{\mathbf{0}}\right)^{-1} \overline{\mathbf{e}}\right]^{2}-\overline{\mathbf{p}}\left(\mathbf{I}-\mathbf{D}_{\mathbf{0}}\right)^{-1} \overline{\mathbf{e}} \\
& \operatorname{cov}\left(T_{\mathbf{1}} T_{k+1}\right)= \overline{\mathbf{p}}\left(\mathbf{I}-\mathbf{D}_{\mathbf{0}}\right)^{-1}\left[\left(\mathbf{I}-\mathbf{D}_{\mathbf{0}}\right)^{-1} \mathbf{D}_{\mathbf{1}}\right]^{k-1}\left(\mathbf{I}-\mathbf{D}_{\mathbf{0}}\right)^{-1} \overline{\mathbf{e}} \\
&-\left[\overline{\mathbf{p}}\left(\mathbf{I}-\mathbf{D}_{\mathbf{0}}\right)^{-1} \overline{\mathbf{e}}\right]^{2}, \quad k \geq 1 .
\end{aligned}
$$

\subsection{Correlation of Interarrival Times of a D-MAP}

The analysis of the correlation decay for interarrival times of a D-MAP is completely analogue to the analysis of Section 3.1. Again, the correlation structure depends on the (a)periodicity of $\left(\mathbf{I}-\mathbf{D}_{\mathbf{0}}\right)^{-1} \mathbf{D}_{\mathbf{1}}$. The geometric decay in the periodic case is determined by the eigenvalue $\mu_{2}$ of $\left(\mathbf{I}-\mathbf{D}_{\mathbf{0}}\right)^{-1} \mathbf{D}_{\mathbf{1}}$ with the largest absolute value, excluding one. An analogue of Theorem 3 can also be stated here in case $\left(\mathbf{I}-\mathbf{D}_{\mathbf{0}}\right)^{-1} \mathbf{D}_{\mathbf{1}}$ is periodic.

\subsection{Limit of the Index of Dispersion for Interarrival Times of a D-MAP}

The dependence among successive interarrival times can be expressed by means of the Index of Dispersion for Intervals (IDI). The IDI, also called the $k$-interval squared coefficient of variation sequence is defined as the sequence $I(k), k \geq 1$, given by

$I(k)=\frac{\operatorname{Var}\left[S_{k}\right]}{k\left(\mathrm{E}\left[T_{1}\right]\right)^{2}}, \quad$ with $\quad S_{k}=T_{1}+\ldots T_{k}$.

It is well known that the following holds :

$$
I(k)=\frac{k \operatorname{cov}\left(X_{1} X_{1}\right)+2 \sum_{j=1}^{k-1}(k-j) \operatorname{cov}\left(X_{1} X_{j+1}\right)}{k\left(\mathrm{E}\left[T_{1}\right]\right)^{2}} .
$$

When the interarrival time distribution is renewal, then $I(k)=c_{1}^{2}$, for all $k \geq 1$, where $c_{1}^{2}$ is the squared coefficient of variation of a single interarrival time (Sriram \& Whitt 1986).

The limit of the IDI is an important measure to characterize the effect of an arrival process on the congestion of a queue in heavy traffic (Iglehart \& Whitt 1970). 
Theorem 6 The limit of the IDI of a D-MAP is given by

$$
\begin{aligned}
\lim _{k \rightarrow+\infty} I(k)= & \lambda^{2}\left\{2 \overline{\mathbf{p}}\left(\mathbf{I}-\mathbf{D}_{\mathbf{0}}\right)^{-1} \mathbf{W}\left(\mathbf{I}-\mathbf{D}_{\mathbf{0}}\right)^{-1} \overline{\mathbf{e}}-\left[\overline{\mathbf{p}}\left(\mathbf{I}-\mathbf{D}_{\mathbf{0}}\right)^{-1} \overline{\mathbf{e}}\right]^{2}\right. \\
& \left.-\overline{\mathbf{p}}\left(\mathbf{I}-\mathbf{D}_{\mathbf{0}}\right)^{-1} \overline{\mathbf{e}}\right\}
\end{aligned}
$$

with $\mathbf{W}$ the fundamental matrix of the Markov Chain $\left(\mathbf{I}-\mathbf{D}_{\mathbf{0}}\right)^{-1} \mathbf{D}_{\mathbf{1}}$, given by

$$
\mathbf{W}=\left[\mathbf{I}-\left(\left(\mathbf{I}-\mathbf{D}_{\mathbf{0}}\right)^{-1} \mathbf{D}_{\mathbf{1}}-\overline{\mathbf{e p}}\right)\right]^{-\mathbf{1}}
$$

PROOF. Follow a similar reasoning as in Theorem 4.

In (Cox \& Lewis 1966) it is shown that the limit of the IDC and the limit of the IDI coincide, i.e. $\lim _{k \rightarrow \infty} C(k)=\lim _{k \rightarrow \infty} I(k)$.

\section{NUMERICAL EXAMPLES}

In this Section we apply the results obtained in Section 3 to the two special output processes considered in Section 2.

\subsection{Example 1}

We consider an ATM multiplexer whose input consist of a number of on/off sources. Both the on and off period are geometrically distributed and while a source is in the on period cells arrive with interarrival time $d$ slots. In (Blondia $\&$ Casals 1992), it has been shown that this superposition can be adequately approximated by means of a D-BMAP. The resulting model for the ATM multiplexer is a $\mathrm{D}-\mathrm{BMAP} / \mathrm{D} / 1 / \mathrm{N}$ queue.

We apply the results described in the previous sections to characterize the correlation structure of the output process. In particular, we illustrate the effect the burstiness of the input sources has on the IDC of the output process. We consider three types of sources, each having the same arrival rate, but with varying burstiness. Clearly type 1 is more bursty than type 2 and type 2 is more bursty than type 3 (see Table).

We let $M=7$ sources enter the multiplexer. The buffer of the multiplexer is assumed to be $N=5$. Figure 2 shows an increasing IDC (and its limit) for an increasing burstiness of the input traffic. 


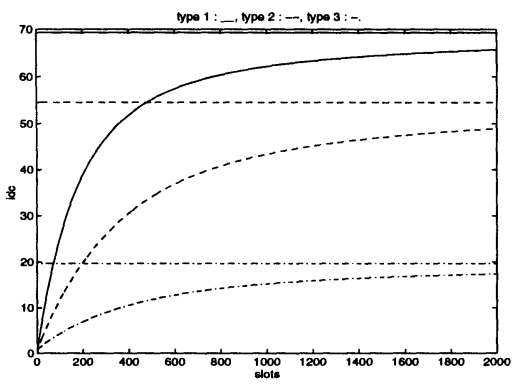

Figure 2 IDC for variable burstiness of input traffic

\begin{tabular}{lllll}
\hline Type & mean on period & mean off period & $\mathrm{d}$ & limit idc \\
\hline type 1 & 120 & 880 & 2 & 69.30 \\
type 2 & 300 & 700 & 5 & 54.56 \\
type 3 & 600 & 400 & 10 & 24 \\
\hline
\end{tabular}

\subsection{Example 2}

Consider a CBR source with interarrival time 4 slots which is mixed with a Poisson traffic stream in a multiplexer. The corresponding matrix of the resulting D-MAP output process has eigenvalue 1 with multiplicity 4 . Figure 3 shows the behavior of the covariance function. In Figure 4 we show a detail of Figure 3, it clearly illustrates Theorem 5 . We distinguish four subsequences of the covariance sequence.

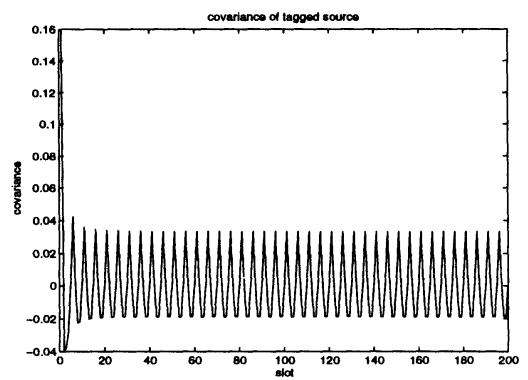

Figure 3 Covariance of a tagged output process

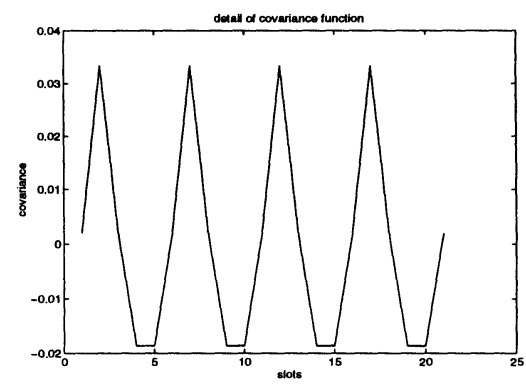

Figure 4 Covariance of a tagged output process : detail 


\subsection{Example 3}

In this example we tag a CBR connection (interarrival time distribution 6 slots) and let it share a multiplexer with Poisson input (= background traffic) with variable arrival rate $\lambda=0, \lambda=0.4$ and $\lambda=0.8$. Figure 5 shows how the covariance changes with the rate of the background traffic $\lambda$. The higher the rate, the flatter the covariance curve, and hence the less important the correlations are.

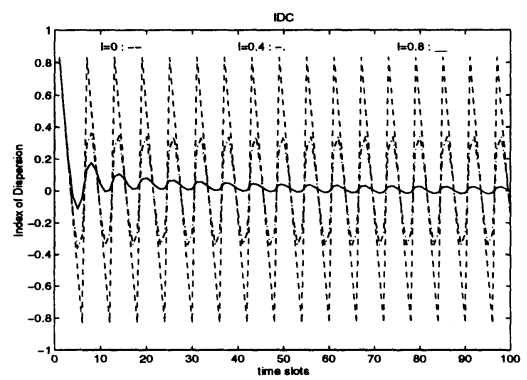

Figure 5 Covariance for variable Poisson background traffic

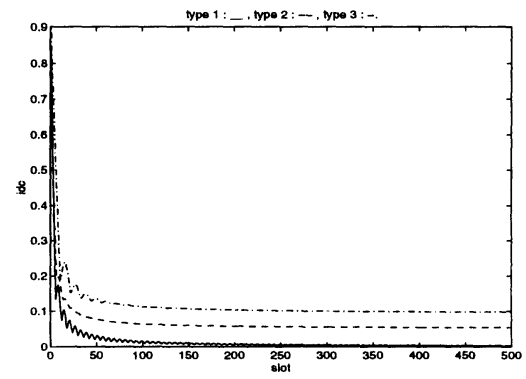

Figure 6 Covariance against variability tagged source

\subsection{Example 4}

In this example we illustrate the impact of the variability of the arrival process of the tagged source on the IDC of the output process. We consider three types of traffic :

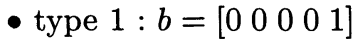

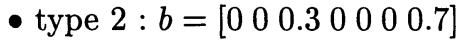

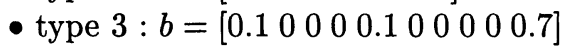

From Figure 6 it follows that the higher the variability of the input stream, the larger the covariance (and its limit) is.

\section{CONCLUSIONS}

In this paper we have investigated the correlation structure of two important models for the output process of an ATM multiplexer. We have given closed formulas for the IDC and IDI and for their limits. Moreover the limiting behavior of the covariance function is also characterized.

These measures of the output process are very useful when investigating endto-end performances in an ATM network. In particular these characteristics 
will be used to describe ATM input traffic to intermediate nodes in future work.

\section{REFERENCES}

Blondia, C. (1993), 'A discrete-time batch Markovian arrival process as BISDN traffic model', Belgian Journal of Operations Research, Statistics and Computer Science 32(3,4), 3-23.

Blondia, C. \& Casals, O. (1992), 'Statistical multiplexing of VBR sources : A matrix -analytic approach', Performance Evaluation 16, 5-20.

Blondia, C. \& Casals, O. (1996), 'Traffic profiles in ATM networks', Telecommunication Systems 5, 49-69.

Blondia, C. \& Theimer, T. (1989), A discrete-time model for ATM traffic, Technical Report PRLB_123_0018_CD_CC/UST_123_0022_CD_CC, RACE document.

Cinlar, E. (1975), Introduction to Stochastic Processes, Prentice-Hall.

Cox, D. \& Lewis, P. (1966), The Statistical Analysis of Series of Events, Methuen, London.

Geerts, F. (1997), Periodic Markov chains and their correlations, available at http://www.luc.ac.be/ fgeerts/index.html.

Heffes, H. \& Lucantoni, D. (1986), 'A Markov modulated characterization of packetized voice and data traffic and related statistical multiplexer performance', IEEE J. on Selected Areas in Communications SAC4(6), 856-868.

Herrmann, C. (1994a), On the analytical model with periodic correlation function of "non-framed-bufferd" VBR video in ATM, in 'IEEE International Telecommunications Symposium', IEEE, Rio de Janeiro.

Herrmann, C. (1994b), VBR video in ATM without frame-buffering: Influence of a periodic correlation function on QOS parameters, in '2nd IFIP Workshop on Performance Modelling and Evaluation of ATM Networks', Bradford, UK.

Iglehart, D. L. \& Whitt, W. (1970), 'Multiple channel queues in heavy traffic II: Sequences, networks and batches', Adv. Applied Prob. 2(2), 353369 .

Kemeny \& Snell (1967), Finite Markov Chains, Van Nostrand.

Lucantoni, D. (1991), 'New results for the single server queue with a batch Markovian arrival process', Stochastic Models 7, 1-46.

Neuts, M. (1979), 'A versatile Markovian arrival process', J. Appl. Prob. 16, 764-779.

Sriram, K. \& Whitt, W. (1986), 'Characterizing superposition arrival processes in packet multiplexers for voice and data', J. on Selected Areas in Communications SAC-4(6), 833-846. 
Call Admission Control (CAC) 